\title{
AN EXTENSION OF THE HEINZ-KATO THEOREM
}

\author{
TAKAYUKI FURUTA
}

(Communicated by Palle E. T. Jorgensen)

Dedicated to Professor Huzihiro Araki on his sixtieth birthday with respect and affection

ABSTRACT. An extension of the Heinz-Kato theorem is given.

In this paper, we shall extend the famous and well-known Heinz-Kato theorem. Please note that a capital letter means a bounded linear operator on a complex Hilbert space $H$.

Theorem A (Heinz-Kato [1, 2]). Let $T$ be an operator on a Hilbert space $H$. If $A$ and $B$ are positive operators such that $\|T x\| \leq\|A x\|$ and $\left\|T^{*} y\right\| \leq\|B y\|$ for all $x, y \in H$, then the following inequality holds for all $x, y \in H$ :

$$
|(T x, y)| \leq\left\|A^{\alpha} x\right\|\left\|B^{1-\alpha} y\right\| \text { for any } \alpha \in[0,1] .
$$

We shall show an extension of Theorem $A$ as follows.

Theorem 1. Let $T$ be an operator on a Hilbert space $H$. If $A$ and $B$ are positive operators such that $\|T x\| \leq\|A x\|$ and $\left\|T^{*} y\right\| \leq\|B y\|$ for all $x, y \in H$, then the following inequality holds for all $x, y \in H$ :

$$
\left|\left(T|T|^{\alpha+\beta-1} x, y\right)\right| \leq\left\|A^{\alpha} x\right\|\left\|B^{\beta} y\right\|
$$

for any $\alpha$ and $\beta$ such that $\alpha, \beta \in[0,1]$ and $\alpha+\beta \geq 1$.

We remark that Theorem A follows by Theorem 1 putting $\alpha+\beta=1$ in Theorem 1, so that Theorem 1 can be considered as an extension of the HeinzKato theorem.

In order to give a proof of Theorem 1, we need the following Theorem B [1, 3] for which there are a lot of proofs-among them, a nice one given in [4].

Theorem B (Löwner-Heinz). If $A \geq B \geq 0$, then $A^{\alpha} \geq B^{\alpha}$ holds for each $\alpha \in[0,1]$.

Also we cite the following obvious lemma.

Lemma. Let $S$ be positive operator. Then:

(i) $(S x, x)=0$ holds for some vector $x$ iff $S x=0$.

(ii) $N\left(S^{q}\right)=N(S)$ holds for any positive real number $q$, where $N(X)$ denotes the kernel of an operator $X$.

Received by the editors May 4, 1992 and, in revised form, June 20, 1992.

1991 Mathematics Subject Classification. Primary 47A30; Secondary 47A63.

Key words and phrases. Positive operator, operator inequality. 
Proof of Theorem 1. First of all, the hypothesis $\|T x\| \leq\|A x\|$ for all $x \in H$ is equivalent to

$$
|T|^{2} \leq A^{2}
$$

Also the hypothesis $\left\|T^{*} y\right\| \leq\|B y\|$ for all $y \in H$ is equivalent to

$$
\left|T^{*}\right|^{2} \leq B^{2} \text {. }
$$

Applying Theorem B to (3) and (4), for any $x, y \in H$ we have

$$
\begin{aligned}
& \left(|T|^{2 \alpha} x, x\right) \leq\left(A^{2 \alpha} x, x\right) \text { for each } \alpha \in[0,1], \\
& \left(\left|T^{*}\right|^{2 \beta} y, y\right) \leq\left(B^{2 \beta} y, y\right) \text { for each } \beta \in[0,1] .
\end{aligned}
$$

Let $N(X)$ denote the kernel of an operator $X$. Let $T=U|T|$ be the polar decomposition of an operator $T$, where $U$ is partial isometry and $|T|=$ $\left(T^{*} T\right)^{1 / 2}$ and $N(U)=N(|T|)$.

In the case $\alpha, \beta \in[0,1]$ such that $\beta>0$ and $\alpha+\beta \geq 1$, we recall the following well-known relation on the polar decomposition of $T$ :

$$
\left|T^{*}\right|^{2 \beta}=U|T|^{2 \beta} U^{*} \text { holds for any } \beta>0 .
$$

Then for all $x, y \in H$ we have

$$
\begin{aligned}
\left|\left(T|T|^{\alpha+\beta-1} x, y\right)\right|^{2} & =\left|\left(U|T|^{\alpha+\beta} x, y\right)\right|^{2}=\left|\left(|T|^{\alpha} x,|T|^{\beta} U^{*} y\right)\right|^{2} \\
& \leq\left\||T|^{\alpha} x\right\|^{2}\left\||T|^{\beta} U^{*} y\right\|^{2}=\left(|T|^{2 \alpha} x, x\right)\left(U|T|^{2 \beta} U^{*} y, y\right) \\
& =\left(|T|^{2 \alpha} x, x\right)\left(\left|T^{*}\right|^{2 \beta} y, y\right) \quad(\text { by (7) }) \\
& \leq\left(A^{2 \alpha} x, x\right)\left(B^{2 \beta} y, y\right) \quad(\text { by (5) and (6)) }
\end{aligned}
$$

for any $\alpha$ and $\beta$ such that $\alpha, \beta \in[0,1]$ and $\alpha+\beta \geq 1$; that is, (2) holds because the result is trivial in the case $\beta=0$.

Hence the proof of Theorem 1 is complete.

Remark 1. In the case $\alpha>0$ and $\beta>0$, the equality in (2) holds for some $x$ and $y$ iff $|T|^{2 \alpha} x$ and $|T|^{\alpha+\beta-1} T^{*} y$ are linearly dependent and $|T|^{2 \alpha} x=A^{2 \alpha} x$ and $\left|T^{*}\right|^{2 \beta} y=B^{2 \beta} y$ hold for some $x$ and $y$ together.

In fact, in the case $\alpha>0$ and $\beta>0$, the equality in the first inequality of (8) holds iff $|T|^{\alpha} x$ and $|T|^{\beta} U^{*} y$ are linearly dependent, that is, $|T|^{2 \alpha} x$ and $|T|^{\alpha+\beta-1}|T| U^{*} y$ are linearly dependent by (ii) of Lemma, namely, $|T|^{2 \alpha} x$ and $|T|^{\alpha+\beta-1} T^{*} y$ are linearly dependent.

The equality in the last inequality of $(8)$ holds iff the equality of $(5)$ and the equality of (6) hold together, that is, $|T|^{2 \alpha} x=A^{2 \alpha} x$ and $\left|T^{*}\right|^{2 \beta} y=B^{2 \beta} y$ hold together for some vector $x$ and $y$ by (i) of Lemma; so the proof of the equality is complete.

Remark 2. The condition $\alpha+\beta \geq 1$ in Theorem 1 is unnecessary if $T$ is a positive operator or invertible operator. This is easily seen in the proof of Theorem 1.

Remark 3. We remark that a condition for which $|T|^{2 \alpha} x$ and $|T|^{\alpha+\beta-1} T^{*} y$ are linearly dependent is equivalent to that $T|T|^{\alpha+\beta-1} x$ and $\left|T^{*}\right|^{2 \beta} y$ are linearly dependent. In fact, the former condition is equivalent to that $|T|^{\alpha} x$ and $|T|^{\beta} U^{*} y$ are linearly dependent as stated in the proof of the equality in the first inequality of (8), and this condition is equivalent to that $U|T|^{\alpha+\beta} x$ and $U|T|^{2 \beta} U^{*} y$ are linearly dependent by (ii) of Lemma and $N(U)=N(|T|)$, that is, $T|T|^{\alpha+\beta-1} x$ and $\left|T^{*}\right|^{2 \beta} y$ are linearly dependent by (7). 


\section{REFERENCES}

1. E. Heinz, Beiträge zur Strüngstheorie der Spektralzerlegung, Math. Ann. 123 (1951), 415-438.

2. T. Kato, Notes on some inequalities for linear operators, Math. Ann. 125 (1952), 208-212.

3. K. Löwner, Über monotone Matrixfunktion, Math. Z. 38 (1934), 177-216.

4. G. K. Pedersen, Some operator monotone function, Proc. Amer. Math. Soc. 36 (1972), 309-310.

Department of Applied Mathematics, Faculty of Science, Science University of TOKYo, 1-3 Kagurazaka, ShinJUKU, TOKYo 162, JAPAN 